\title{
Hospitalizaciones asociadas al diagnóstico de fibrilación auricular en Chile. Tendencia creciente en la última década.
}

\author{
Mario Ortiz ${ }^{1}$, Elena Ortiz ${ }^{2}$, Raimundo Morris ${ }^{1}$, René Asenjo $^{1}$, Mauricio Cereceda ${ }^{1}$. \\ 1) Departamento Cardiovascular, Hospital Clínico, Universidad de \\ Chile. \\ 2) Dirección de Salud Rural, Servicio de Salud de Aysén.
}

Antecedentes: Publicaciones internacionales señalan que las hospitalizaciones por fibrilación auricular (FA) están aumentando, lo que tiene importantes implicaciones para la salud pública y el cardiólogo. En Chile no se dispone de mayor información sobre el problema. Objetivo: Estudiar las hospitalizaciones asociadas al diagnóstico de FA en Chile.

Métodos: Los egresos hospitalarios con el diagnóstico de FA fueron obtenidos del Ministerio de Salud (código I-48 FA) de la Clasificación Internacional de Enfermedades. Hubo datos disponibles solo para los años 2002 al 2007; se analizó género, grupos etarios y regiones del país. Las tasas $(\mathrm{T})$ de egresos hospitalarios con este diagnóstico por 10.000 habitantes $(\mathrm{H})$ fueron calculadas en base a la población proyectada del último censo nacional para los años señalados. Para el análisis estadístico se utilizó el test de proporsiones de Z. Resultados: La T total de egreso hospitalario asociada al diagnóstico de FA el año 2002 fue de 2,2/10.000 H la que aumentó gradualmente hasta una $\mathrm{T}$ de 2,8/10.000 $\mathrm{H}$ el año 2007 ( $\mathrm{P}<0,001)$. Las $\mathrm{T}$ de hombres y mujeres el año 2002 fueron de 2,2 y 2,1 y aumentaron hasta 2,8 y $2,7 / 10.000 \mathrm{H}$ el 2007 , respectivamente $(\mathrm{P}<0,001)$. En el grupo etario $\leq 40$ años no se observaron mayores variaciones de las T entre los años 2002 y $2007(\mathrm{P}<0,01)$. En los mismos períodos las T en pacientes de 40-64 años fueron de 2,9 y 3,6/10.000 H, entre 65-79 años 14,2 y $18,5 / 10.000 \mathrm{H}(\mathrm{P}<0,001)$, mientras que en $\geq 80$ años las $\mathrm{T}$ fueron de 32,4 y $43,7 / 10.000 \mathrm{H}$ respectivamente $(\mathrm{P}<0,0001)$. En las regiones I-II-III, las $\mathrm{T}$ no presentaron variaciones, pero en las regiones $\mathrm{X}$-XII-XII las $\mathrm{T}$ aumentaron gradualmente en el periodo estudiado (2,3 y $3,5 / 10.000 \mathrm{H}$ los años 2002 y 2007, respectivamente). Conclusiones: Este trabajo muestra que en nuestro país, las $\mathrm{H}$ por FA muestran una tendencia creciente, con un incremento de $27 \%$ en el periodo estudiado, especialmente en $\geq 65$ años, y en las regiones del sur del país. Se requiere profundizar en las causas de este aumento. 


\section{Hospitalization for atrial fibrillation in chile. a growing tendency in the last decade}

Background: Hospitalization for atrial fibrillation $(\mathrm{AF})$ is reported to be increasing worldwide, becoming a cardiovascular health problem. Scarce data on this subject is available in Chile.

Aim: To describe hospitalizations related to $\mathrm{AF}$ in Chile.

Method: The discharge diagnosis of AF (ICD I48)was obtained form the Ministry of Health reports. Only data from 2002 through 2007 was available. Gender, age and location (regions) were analyzed. A projection derived form the last population census was used to estimate rates per 10.000 people discharged with a diagnosis of $\mathrm{AF}$

Results: The rate of discharges with a diagnosis of AF increased from 2.2 per 10.000 in 2002 to 2.8 per 10.000 in 2007. Male and female rates were 2.2

\section{Introducción:}

La fibrilación auricular es la arritmia sostenida más común encontrada en la práctica clínica estimándose que afecta al 1-2\% de la población general y se considera muy probable que esta cifra aumente significativamente en los próximos 50 años ${ }^{1}$. La arritmias está asociada con un bien documentado aumento de la morbilidad y mortalidad entre los cuales el accidente vascular cerebral embólico es una de las complicaciones mas relevantes ${ }^{1,2}$. En concordancia con estos datos, las hospitalizaciones por fibrilación auricular han presentado un aumento progresivo en países desarrollados con las consecuentes implicaciones de costo para los sistemas de salud ${ }^{3,4}$.

En Chile, un país con algunos indicadores demográficos y de salud similares al de países desarrollados, es posible suponer que también estamos enfrentados a un aumento de las cifras de fibrilación auricular y morbimortalidad asociada y, en consecuencia, a un incremento en las hospitalizaciones relacionadas con esta arritmia. No obstante, en nuestro país no se dispone de mayor información acerca de la realidad nacional sobre el problema.

El objetivo del presente trabajo fue estudiar las hospitalizaciones asociadas al diagnóstico de fibrilación auricular en Chile.

\section{Métodos:}

a) Los datos fueron obtenidos del Departamento de Es- and 2.1 respectively in 2002, increasing to 2.8 and 2.7 in 2007. The rate of AF discharge remained approximately constant for those under 40 years of age. In contrast, the corresponding rates for 2002 and 2007 increased from 2.9 to 3.6 for those aged 40-64, 14.2 to 18.5 for those aged $65-79$ and 32.4 to 43.7 for those over 80. AF discharge rates did no vary in Regions I to III while they increased from 2.3 to 3.5 in regions $\mathrm{X}$ to XII.

Conclusion: The rate of discharge with a diagnosis of $\mathrm{AF}$ has increased in Chile approximately $25 \%$ from 2002 to 2007. This phenomenon is more marked in those over 65 years of age and those living in the southern regions of the country

Keywords: atrial fibrillation, hospitalization, elderly.

tadísticas e Información en Salud del Ministerio de Salud ${ }^{5}$. La base de datos de este Departamento fue revisada específicamente en cuanto a los egresos hospitalarios con el diagnóstico de fibrilación auricular mediante el análisis del código I-48 (fibrilación auricular) de la Clasificación Internacional de Enfermedades

b) La información de egresos hospitalarios con el diagnóstico de esta arritmia, incluyendo a todo el país, fue disponible sólo para los años 2002 a 2007 y constituye el período analizado en el presente trabajo.

c) Los egresos hospitalarios con el diagnóstico de fibrilación auricular fueron estudiados en relación a grupos etarios, género y regiones de todo el país.

d) Las tasas de egresos hospitalarios con este diagnóstico por 10.000 habitantes, fueron calculadas en base a la población proyectada del último censo nacional (6) para los años señalados.

e) Para el análisis estadístico de las tendencias observadas, se utilizó el test de proporciones de Z. El análisis fue realizado con el programa STATA 10,0.

\section{Resultados:}

Tasas totales de egresos hospitalarios con el diagnostico de fibrilación auricular

La tasa total de egresos hospitalarios con el diagnóstico de fibrilación auricular en los años 2002 y 2003 fue de 2,2/10.000 habitantes y en los años 2004 y 2005 de 
2,4/10.000 habitantes. En los años 2006 y 2007 las tasas fueron de 2,6 y 2,8/10.000 habitantes, respectivamente (Fig.1) $(\mathrm{P}<0,001)$.

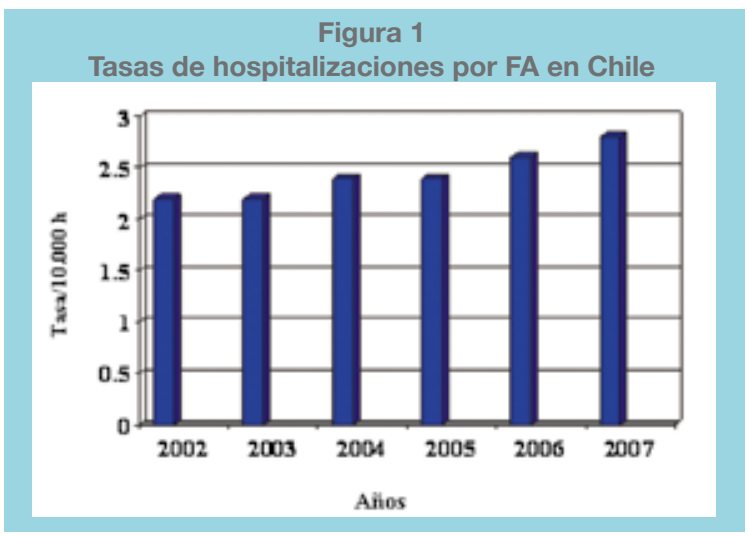

Tasas de egresos hospitalarios con el diagnóstico de fibrilación auricular y género

La tasa de egreso hospitalario de los hombres el año 2002 fue de 2,2/10.000 habitantes y salvo un leve descenso el año $2003(2,1 / 10.000)$ presentó un aumento gradual con una tasa de 2,8/10.000 habitantes el año $2007(\mathrm{P}<0,001)$. La tasa de las mujeres el año 2002 fue de 2,1/10.000 habitantes y en los años 2003 y 2004 fue de 2,2/10.000 y 2,4/10.000 respectivamente. El año 2005 la tasa fue menor (2,2/10.000 habitantes), pero en los años siguientes se incrementó nuevamente hasta una tasa de 2,7/10.000 habitantes el año 2007 (Fig. 2) (P<0,001).

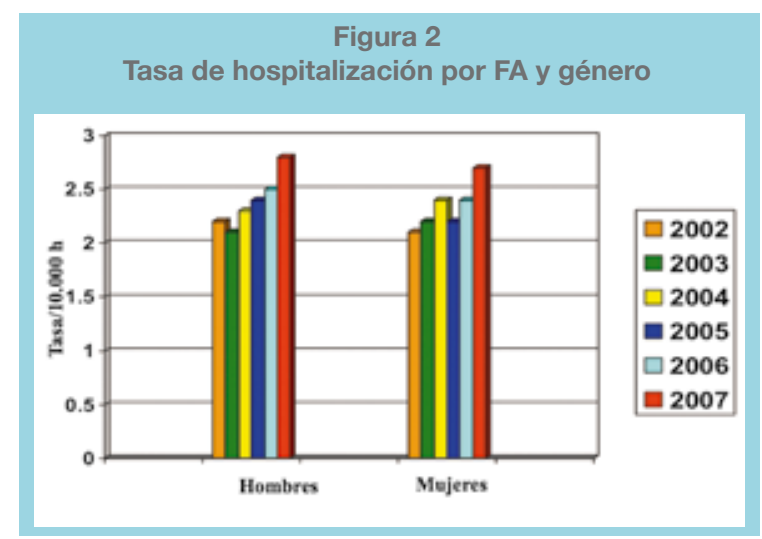

Tasas de egresos hospitalarios con el diagnóstico de fibrilación auricular y edad

El año 2002 la tasa en pacientes de edad $\leq 40$ años fue de 0,2/10.000 habitantes y se mantuvo sin mayores variaciones con una tasa de 2,3/10.000 habitantes el año 2007
(Fig. 3). La tasa de pacientes de 40-64 años el año 2002 y 2003 fue de 2,9/10.000 habitantes y de 3,2/10.000 en los años 2004 y 2005. Las tasas fueron mayores en los 2 años siguientes alcanzando una cifra de 3,6/10.000 habitantes el año $2007(\mathrm{P}<0,01)$. En pacientes de 65-79 años el año 2002 la tasa fue de 14,2/10.000 y se mantuvo sin mayores variaciones hasta el año 2004 (14,7/10.000). Posteriormente se observó un aumento gradual hasta una tasa de $18,5 / 10.000$ habitantes el año $2007(\mathrm{P}<0,001)$. En el grupo etario $\geq 80$ años la tasa fue de 32,4/10.000 el año 2002 y en los años siguientes presentó un aumento progresivo, particularmente en los últimos 3 años del período estudiado, con una tasa de 43,7/10.000 habitantes el año 2007 $(\mathrm{P}<0,0001)$ (Fig. 3).

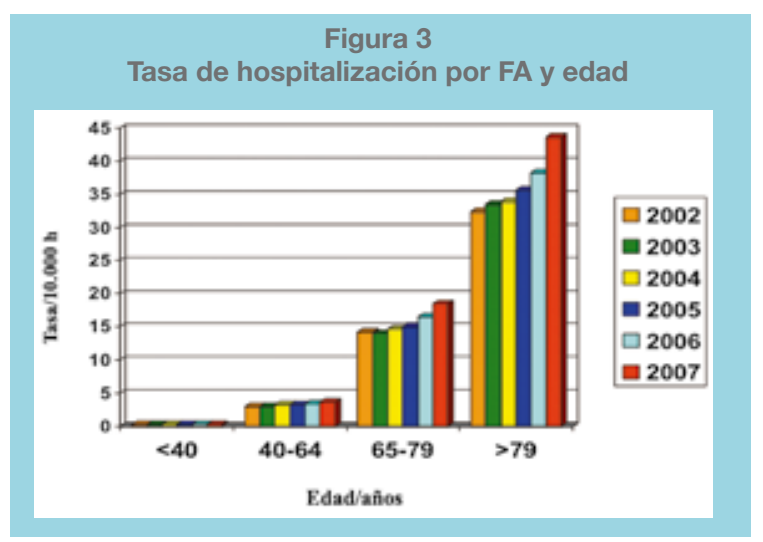

Tasas de egresos hospitalarios con el diagnóstico de fibrilación auricular y regiones del país

En las regiones del norte del país, I-II-III, la tasa fue de 1,3/10.000 habitantes el año 2002 y no presentó mayores variaciones en los años siguientes con una tasa similar el año 2007 (Fig. 4). En la IV y V región la tasa fue de 2,0/10.000 habitantes el año 2002. Esta tasa disminuyó levemente en los 2 años siguientes y aumentó a partir del año 2005 con una tasa de 2,5/10.000 habitantes en los años 2006 y 2007 ( $\mathrm{P}<0,001)$. La tasa en la región Metropolitana el año 2002 fue de 2,3/10.000 habitantes y no presentó mayores variaciones en los 4 años siguientes con una tasa levemente mayor el año 2007 de 2,5/10.000 $(\mathrm{P}<0,035)$ habitantes. En las regiones VI-VII la tasa fue de 1,9/10.000 habitantes el año 2002 y de 2,0/10.000 habitantes el año 2003. Las tasas fueron similares los 2 años siguientes y aumentaron a 2,4 y 3,1/10.000 habitantes en los años 2006 y 2007 ( $\mathrm{P}<0,0001)$, respectivamente. En las regiones VIII-IX la tasa fue de 2,3/10.000 habitantes el año 2002 y presentó un aumento progresivo en los años siguientes hasta una tasa de 3,6/10.000 habitantes el año 
2007 ( $\mathrm{P}<0,0001)$. En las regiones del extremo sur del país, X-XI-XII, la tasa fue de 2,3/10.000 habitantes el año 2002 y 2003. Las tasas fueron mayores en los 3 años siguiente alcanzando un cifra de 3,5/10.000 habitantes el año 2007 $(\mathrm{P}<0,0001)$ (Fig. 4).

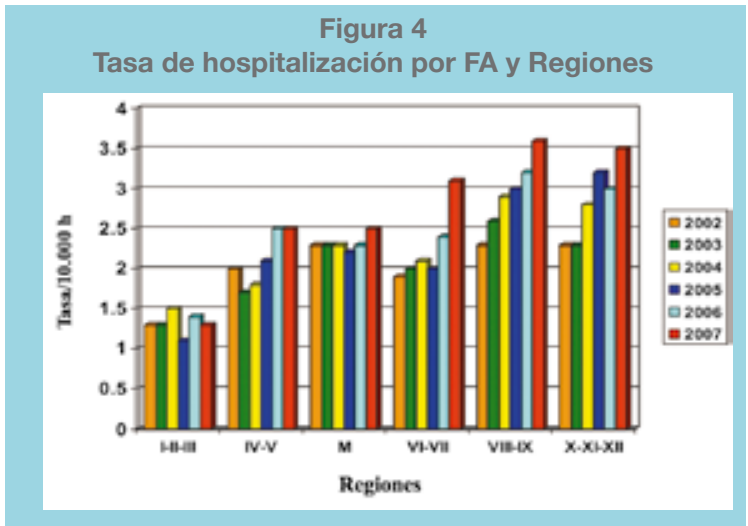

\section{Discusión:}

Este trabajo muestra que la tasa de hospitalización a nivel nacional, asociada al diagnóstico de fibrilación auricular, aumentó de 2,2/10.000 habitantes el año 2002 a 2,8/10.000 el año 2007, lo que constituye un aumento de $27 \%$ en los 6 años estudiados. Estos datos representan la primera información nacional sobre las hospitalizaciones en relación a la fibrilación auricular y su incremento en la última década. En Norteamérica, el aumento de las hospitalizaciones con el diagnóstico de fibrilación auricular fue reportado hace 2 décadas encontrándose que ellas prácticamente se duplicaron entre los años 1985 y $1999^{3}$. Este aumento se observó tanto en hombres (desde 76 a 154/10.000 habitantes), como en mujeres (desde 75 a 166/10.000 habitantes). Nuestros resultados son concordantes con esos datos en mostrar un aumento, aunque de menor magnitud, en hombres y mujeres con tasas también similares en ambos géneros. También es concordante que la prevalencia de hospitalizaciones por fibrilación auricular en nuestro medio es claramente mayor en los sucesivos grupos etarios y en todos los años estudiados. El incre- mento de las tasas de hospitalización a lo largo del tiempo es diferente, con solo un leve aumento en los pacientes de 40 a 65 años y un apreciable incremento en pacientes de 80 o más años (Figura 3).

En nuestros resultados llama la atención que las tasas de hospitalización por fibrilación auricular son mayores en las regiones del sur del país (VIII a la XII) en comparación a las regiones del norte (I-II-III) donde, además, no se observó aumento de las tasas (1,3/10.000 habitantes en las regiones I-II-III, años 2002 y 2007, y 2,3/10.000 habitantes en las regiones X-XI-XII el año 2002 y 3,5/10.000 habitantes el año 2007). Trabajos realizados en Europa ${ }^{7,8}$ ${ }^{9} \mathrm{y}$ uno en Australia (10) han mostrado una modesta variación estacional en la ocurrencia de fibrilación auricular con tasas de hospitalización algo mayores en los meses de invierno. Los factores causales implicados en esos hallazgos no están dilucidados y han sido controversiales ${ }^{10,11}$. ${ }^{2}$. Se ha señalado que efectos directos o indirectos de las bajas temperaturas sobre el sistema cardiovascular pueden favorecer la ocurrencia de fibrilación auricular pero también el aumento de comorbilidades más prevalentes en los meses de invierno o climas fríos, podría explicar el aumento de las hospitalizaciones ${ }^{2}$.

Las tasas de hospitalización del presente trabajo son inferiores a las reportadas en norteamérica ${ }^{3,12,13}$ y europa ${ }^{4}$ lo que muy probablemente se debe a subregistro, en nuestro medio, del diagnóstico de fibrilación auricular entre los diagnósticos incluidos en la hoja de egreso hospitalario. No obstante, aun con esta limitación, estimamos que estos datos aportan una información de interés y cobertura nacional sobre el problema, hasta ahora no disponible en nuestro país.

En conclusión, el presente trabajo muestra que las hospitalizaciones asociadas al diagnóstico de fibrilación auricular en Chile presentaron un aumento relevante entre los años 2002 y 2007, lo cual tiene importantes implicaciones para la salud pública y la cardiología nacional. Mayores estudios son necesarios para conocer la realidad clínicoepidemiológica de la fibrilación auricular en Chile.

\section{Referencias:}

1.- THE TASK FORCE FOR THE MANAGEMENT OF ATRIAL FIBRILLATION OF THE EUROPEAN SOCIETY OF CARDIOLOGY. Guidelines for the Management of Atrial Fibrillation. Europace 2010;12:1360-1420.

2.- GREENLEE T, VIDAILLET H. Recent progress in the epide- miology of atrial fibrillation Curr Opin Cardiol 2007;20:7-14.

3.- WATTIGNEY WA, MENSAH GA, CROFT JB. Increasing Trends in Hospitalization for Atrial Fibrillation in the United States, 1985 Through 1999. Implication for Primary Prevention. Circulation 2003;108:711-716.

4.- $\quad$ FRIGERD J, BUCH P, SCHARLING H, GADSBPHIOLL N, JENSEN GB. Raising rates of hospital admisions for atrial fibrillation. Epidemiology 2003;14: 666-72. 
Hospitalizaciones asociadas al diagnóstico de fibrilación auricular en Chile. Tendencia creciente en la última década.

Ortiz $M$, et al.

5.- $\quad$ http://deis.minsal.cl/index.asp

6.- www.ine.cl

7.- $\quad$ FROST L, JOHNSEN SP, PEDERSEN L, HUSTED S, ENGHOLM G, SØRENSEN HT, et al. Seasonal variation in hospital discharge diagnosis of atrial fibrillation: a population-based study. Epidemiology 2002;13: 211-215.

8.- MOUTZOURIS DA, HASSID VJ: Seasonal variations of atrial fibrillation: further supportive evidence. Epidemiology 2003; 14: 127.

9.- GŁUSZAK A, KOCOŃ S, ZUK K, ALJABALI P, GLUZA A, SIWEK K. Episodes of atrial fibrillation and meteorological conditions. Kardiol Pol 2008; 66: 958-963.

10.- KIU A, HOROWITZ JD, STEWARD S: Seasonal variation in
AF-related admission to a coronary care unit in a "hot" climate: fact or fiction? J Cardiovasc Nurs 2004;19:138-141.

11.- UPSHUR RE, MOINEDDIN R, CRIGHTON EJ, MAMDANI M. Is there a clinically significant seasonal component to hospital admissions for atrial fibrillation? BMC Health Services Research 2004; 4: 5-11.

12.- KHAIRALLAH F, EZZEDINE R, GANZ L, LONDON B, SABA S. Epidemiology and Determinants of Outcome of Admissions for Atrial Fibrillation in the United States from 1996 to 2001 Am J Cardiol 2004; 94: 500-504.

13 HUMPHRIES KH, JACKEVICIUS C, GONG Y, SVENSEN L, COX J, TU JV, et al Population rates of hospitalization for atrial fibrillation/flutter in Canadá. Can J Cardiol 2004; 20: 869-76 